\title{
Episiotomy Closure After Vaginal Delivery - A Prospective, Single Centre Study Assessing a Novel Fast-Absorbable, Monofilament Suture in Daily Practice
}

\author{
Petra Baumann ${ }^{1, *}$, Michael Weigel ${ }^{2}$ \\ ${ }^{1}$ Department Medical Scientific Affairs, Aesculap, Tuttlingen, Germany \\ ${ }^{2}$ Department of Obstetrics \& Gynaecology, Leopoldina Hospital, Schweinfurt, Germany
}

Email address:

petra.baumann@aesculap.de (P. Baumann)

${ }^{*}$ Corresponding author

To cite this article:

Petra Baumann, Michael Weigel. Episiotomy Closure After Vaginal Delivery - A Prospective, Single Centre Study Assessing a Novel FastAbsorbable, Monofilament Suture in Daily Practice. Journal of Surgery. Vol. 9, No. 3, 2021, pp. 121-127. doi: 10.11648/j.js.20210903.15

Received: April 8, 2021; Accepted: April 29, 2021; Published: May 26, 2021

\begin{abstract}
This is the first assessment on the performance of a new fast-absorbable monofilament suture for episiotomy closure under daily clinical routine. The study was designed as a prospective, single centre, observational study including 50 women. Satisfaction and pain using the visual analogue scale was rated at discharge and eight weeks post-partum. Handling characteristics of the suture were assessed intrasurgically by the obstretricians using a 5-point Likert scale. Adverse events and wound healing outcome was monitored for eight weeks after delivery. Perineal pain rate as well as the pain level in different categories strongly decreased from discharge to eight weeks post-partum. Patient satisfaction was high, $84.4 \pm 18.97$ and $91.2 \pm 16.86$ at discharge and eight weeks after delivery, respectively. In total, $50 \%$ of the women had sexual intercourse 10 weeks post-partum. Suture handling was rated good to excellent by the obstretricians. Wound healing assessment indicated an excellent outcome. No wound dehiscence, wound infection, tissue reaction, allergic or inflammatory reaction occurred. Neither need for resuturing of the wound nor intrasurgical suture rupture were reported. Findings indicate that the clinical performance of novel, fast-absorbable, monofilament suture is equivalent to current sutures used for episiotomy closure and can be regarded as a viable alternative to rapid, absorbable multifilaments.
\end{abstract}

Keywords: Episiotomy, Perineal Repair, Perineal Pain, Suture, Wound Healing, Patient Satisfaction

\section{Introduction}

An episiotomy is a surgical incision which is made in the perineum to enlarge the vaginal opening for birth and is one of the most commonly performed procedures in obstetrics [13]. There are three types of episiotomies which can be performed: midline, mediolateral and lateral. Due to the high complication rate of the lateral type, this incision is nowadays obsolete. Morbidity associated with childbirth may effect women's physical, psychological and social wellbeing, both in the immediate and long-term postnatal period $[4,5]$. The type of the suture material, operator skills and the technique used for repair are the three main factors that influence the short and long term outcome of perineal repair $[6,7]$.

Approximation using the continuous suture technique for episiotomies is associated with significantly less short-term perineal pain, higher patient satisfaction, less analgesics use and substantially lower suture removal compared to the interrupted technique [8-10]. In addition, the continuous technique is faster and more cost-effective than the interrupted technique [8]. Rapidly-absorbable, braided synthetic sutures are reported to be superior to monofilament suture or other synthetic materials with slower absorption profile in regard to perineal pain and wound healing [6]. Application of continuous, synthetic, multifilament, rapidly absorbable sutures using polyglactin 910 (Vicryl ${ }^{\circledR}$ Rapide) has become the common practice for episiotomies $[6,11]$. Vicryl $^{\mathbb{B}}$ Rapide was approved in 1987. It gives wound support up to 14 days post-implantation and has a total absorption time of 42 days. Several publications are available using Vicryl ${ }^{\circledR}$ Rapide for episiotomy repair $[6,12-20]$. 
Comparison between standard absorbable polyglactin 910 and rapid, absorbable polyglactin 910 suture showed a significant difference in regard to pain at 10 days post-partum and a lower rate of suture removal in favour of rapid absorbable polyglactin 910 suture [7, 20]. Many hospitals choose rapide-absorbable polyglactin 910 suture as a first line suture material for episiotomy repair.

To date only three trials have compared an absorbable monofilament with an absorbable multifilament suture for episiotomy repair of $[12,15,21]$. Based on their filament structure, braided multifilaments carry a higher risk for wound irritation and wound infection due to their capillary properties [12].

In 2006, Dencker et al. firstly studied in a randomized controlled trial the clinical outcome of a monofilament glycomer (Biosyn) versus a multifilament polyglycolic acid suture (Dexon) for suturing of lacerations and episiotomies following vaginal delivery [21]. Perineal healing, discomfort levels and pain did not differ between the suture groups until three days post-partum, but perineal wound healing was significantly better in the multifilament group 8-12 weeks after delivery. In addition, at the 8-12 weeks follow-up, higher levels of discomfort and pain were reported by women sutured with a monofilament, which was due to non-absorbed stitches. Authors conclude that the disadvantages seen with the monofilament suture might be due to longer absorption time (90-110 days) compared to polyglycolic acid (60-90 days) and that the allocation to a more rapid, absorbable suture would reduced the necessity of suture removal and would be of higher benefit for women with the need of suturing after childbirth.

A randomised, controlled study performed by Kokanali in 2011 aimed to find an optimal suture material for episiotomy repair, and showed comparable results using either fastabsorbable polyglactin 910 (Vicryl Rapide) or an absorbable, monofilament suture (polyglycolide-co-caprolactone) [15]. Authors found similar perineal pain scores in the short-term and long-term post-partum period as well as during sexual intercourse with regard to the filament structure used for repair. Furthermore, no difference between the suture groups was observed according to the rate of suture removal and incomplete wound healing. Neither infections nor haematomas were present [15].

The MOVE-trial, a randomized controlled trial published in 2017, determine which suture material (absorbable, monofilament, polyglecaprone 25 (Monocryl) or fastabsorbable, braided, polyglactin 910 (Vicryl Rapide) is superior for intracutaneous closure of the skin in mediolateral episiotomies [12]. Equal perineal pain rates were reported in both suture groups, whereas the wound dehiscence rate was significantly lower in the monofilament group at 10 days and three months after delivery. Authors conclude that both suture materials can be considered for use, but an absorbable monofilament suture might be favourable over a fastabsorbable, braided multifilament due to a decreased wound dehiscence rate [12].

In June 2016, a new suture was released, based on a fast- absorbable, monofilament suture (Monosyn ${ }^{\circledR}$ Quick) made from a triblock copolymer comprising glycolide (72\%), $\varepsilon$ caprolactone (14\%) and trimethylene carbonate (14\%). Upon implantation, this surgical suture retains $70 \%-80 \%$ of its initial tensile strength after 5 days, and 20\% - 30\% tensile strength after 10 days. The tensile strength is completely lost after 14 - 21 days post implantation and suture absorption time is 56 days.

The purpose of the present study was to assess for the first time the clinical performance of a new, fast-absorbable, monofilament suture for episiotomy closure and to compare the outcome with literature data available for fast-absorbable, braided multifilaments used for perineal repair.

\section{Method}

\subsection{Study Design, Participants and Ethical Considerations}

We designed a prospective, single centre, single-arm consecutive study to evaluate perineal pain, wound healing outcome, intrasurgical handling, and postsurgical patients satisfaction after episiotomy repair. The trial was registered before the start of recruitment (November 28, 2017) in ClinicalTrials.gov under the registration number NCT03355001. Ethics approval was obtained by the Ethical Committee of the University of Würzburg, Germany, ref. no. $167 / 17$ on 12 December 2017. All women were informed verbally and written informed consent was obtained before treatment. Episiotomy repairs were performed by different obstretricians. Monosyn ${ }^{\circledR}$ Quick suture (B. Braun Surgical S. A. Rubi, Spain) was applied in a three-layer technique to approximate the vaginal mucosa, the perineal muscle and the skin and the suture technique was chosen by the obstretrician. The inclusion criteria were as follows: primiparous as well as multiparous women with or without a previous perineal surgery undergoing an episiotomy in routine clinical settings, both women with a spontaneous or surgical delivery, age $\geq$ 18 years. Specific exclusion criteria were not selected. Women were treated according to the local standards. After enrolment, the episiotomy was performed, women were examined at discharge and followed up at eight weeks \pm two weeks after delivery. The data were collected in a paper based Case Report Form (CRF) and transferred in an online database by the clinic's study nurse.

\subsection{Outcomes}

Suture handling was intrasurgically evaluated after each episiotomy repair by the obstretrician using a questionnaire including different dimensions (knot security, tensile strength, knot run down, tissue drag and stiffness). Each dimension was rated on a five-point Likert-type scale using the following categories: excellent, very good, good, satisfied and poor. Additionally, intrasurgical complications of the suture material such as thread rupture, knots in the thread, bended thread, defect in needle-thread attachment were reported.

Patient satisfaction with the repair was recorded using a 
Visual Analogue Scale, VAS (0 low - 100 excellent) eight weeks after delivery. Presence of perineal pain in different positions (in repose, when sitting, when walking, during urinating and during defecating) was evaluated by the women using the VAS ( 0 no pain - 100 maximum pain unimaginable) at discharge and eight weeks post-partum. In addition, eight weeks after episiotomy repair the parturients were questioned by the study nurse if sexual intercourse had been happened or not and the same VAS scale was used to quantify the score of pain during sexual intercourse.

Complications such wound dehiscence, wound infection, seroma, fistula, abscess formation, haematoma, tissue reaction, allergic reaction, inflammation and the need for suture removal due to incomplete or non-absorbed suture occurring within eight weeks post-partum were noted. At the postnatal visit after eight weeks of delivery the obstretrician rated the perineal wound healing using the VAS ( 0 poor 100 excellent).

\subsection{Statistical Methods and Sample Size}

All women receiving the fast-absorbable monofilament suture for episiotomy repair without any protocol violation were included in the per-protocol analysis. A sample size of 50 patients was considered to be appropriate to detect substantial deviations in comparison to Vicryl ${ }^{\circledR}$ Rapide, which is regarded as the market reference. The results from the present study were analysed descriptively and compared with corresponding data from the literature $[7,9,10,14,19$, 20]. If the data of this study correspond to published data of Vicryl $^{\circledR}$ Rapide, the suture material Monosyn ${ }^{\circledR}$ Quick was considered as equivalent. For comparison of our study results with corresponding ranges from the literature, 95\% confidence intervals (Agroforestry-Coull method) were applied. Confidence intervals allow to establish ranges for each analysed parameter. Statistical analysis was performed using SAS software version 9.4 (SAS Institute Inc., Cary, NC, USA). Variables with metric or ordinal scale were summarised with number of patients (N), minimum (Min), maximum (Max), median, mean, and standard deviation (SD). Categorical variables were summarised by category to absolute $(\mathrm{N})$ and relative (\%) frequency, with missing values building an own category. Missing data were analysed as such and were not replaced by estimates. T-test was used for statistical comparisons.

References gained from the literature with their 95\% confidence intervals used for comparison:

Postsurgical complications:

1) Wound dehiscence 10 days after post-partum (0.059 [0.046; 0.075])

2) Re-suturing up to 3 months after delivery (0.013 [0.007; 0.023])

3) Suture removal due to wound problems (infection, gaping wound, residual material requiring removal) up to three months after delivery $(0.061[0.049 ; 0.074])$.

4) Remaining suture material six weeks post-partum $(0.039[0.026 ; 0.058])$

Perineal pain:
1) Short-term perineal pain after $24-48 \mathrm{~h}, 10$ days postpartum (resp. $0.56[0.52 ; 0.60]$ and $0.31[0.28 ; 0.33]$ )

2) Long term perineal pain 3 months post-partum $(0.024$ $[0.012 ; 0.044])$

Table 1. Pain level in different categories with their $95 \%$ confidence intervals used for comparison.

\begin{tabular}{lll}
\hline & 24h-48h post-partum & 10 days post-partum \\
\hline in repose, & $0.43[0.38 ; 0.47]$ & $0.16[0.13 ; 0.20]$ \\
during walking, & $0.67[0.62 ; 0.71]$ & $0.33[0.29 ; 0.37]$ \\
during sitting, & $0.70[0.65 ; 0.74]$ & $0.40[0.35 ; 0.45]$ \\
when urinating, & $0.53[0.48 ; 0.58]$ & $0.29[0.25 ; 0.34]$ \\
when defecating & $0.37[0.30 ; 0.43]$ & $0.31[0.27 ; 0.35]$ \\
\hline
\end{tabular}

1) Return to sexual intercourse 3 months post-partum $(0.86[0.80 ; 0.90])$

2) Time to start with sexual intercourse (days) (47.4 [46.0; 48.8])

3) Patient satisfaction with the repair 3 months postpartum $(0.81[0.78 ; 0.83])$

\section{Results}

\subsection{Recruitment and Follow-up Examination}

Between January 2018 and April 2018 in total 50 women were recruited and examination at eight weeks post-partum was completed in June 2018. All women received Monosyn ${ }^{\circledR}$ Quick suture for episiotomy repair. Documentation of the discharge visit was available of forty-seven parturients, data of three women were not recorded at this time point. Fortyeight women were successful contacted and examined eight weeks post-partum, two were lost to follow-up due to language barriers (Figure 1).

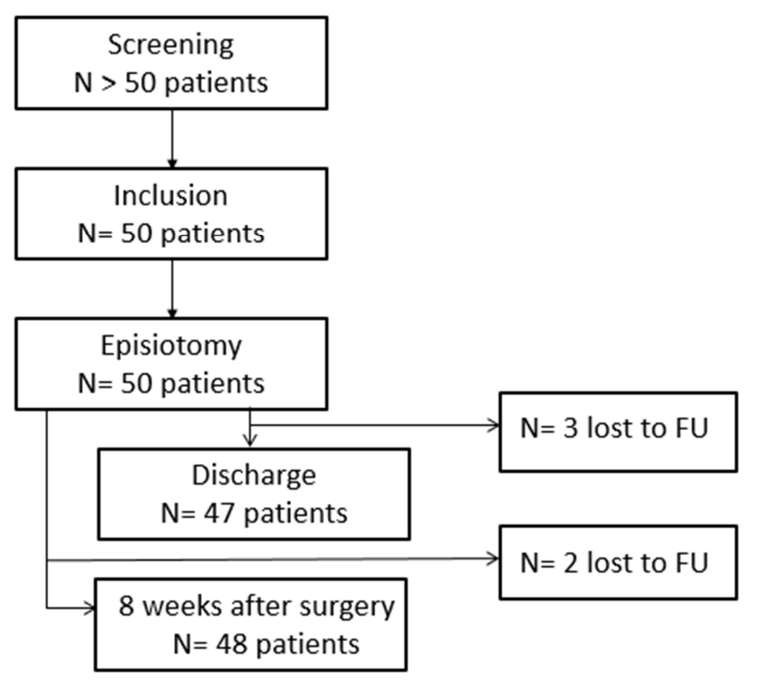

Figure 1. Flow Chart of the study.

\subsection{Demographics and Other Baseline Characteristics}

Demographics and baseline characteristics are listed in table 2 . The population consisted of $43(86 \%)$ primiparous and $7(14 \%)$ multiparous women. A previous perineal surgery was recorded in 6 women (12\%) and 52 newborns including 
two twins were delivered. Epidural anaesthesia was received by 20 women $(40 \%)$, mode of delivery was spontaneous in 47 cases $(94 \%)$ and vacuum support was needed in $6 \%$ of the population. Table 3 shows that obstretricians preferred the continuous suture technique to approximate the different tissue layers (88-98\%) in combination with a suture USP size $4 / 0$ fixed to a DS $19 \mathrm{~mm}$ needle (100\%). Only in one case, USP size $3 / 0$ was chosen. Amount of suture material used for the approximation of the different tissue layers was $10 \pm 4.4$ threads and time to close the episiotomy was recorded with $17.82 \pm 6.9$ minutes. Women stayed in the clinic for $3 \pm 2.7$ days.

Table 2. Demographic and baseline data of 50 women undergoing episiotomy repair with Monosyn ${ }^{\circledR}$ Quick.

\begin{tabular}{lll}
\hline & N & Median (range) \\
\hline Women & 50 & \\
Maternal age (years) & 50 & $29(20-39)$ \\
Weight $(\mathrm{kg})$ before delivery & 50 & $77(52.8-120)$ \\
Height $(\mathrm{cm})$ & 50 & $166(156-180)$ \\
BMI $\left(\mathrm{kg} / \mathrm{m}^{2}\right)$ before delivery & 50 & $27.51(19.83-44.14)$ \\
Gestation week & 50 & $40(31-42)$ \\
& & \\
Newborn & 52 & \\
Birth weight $(\mathrm{g})$ & 52 & $3330(1745-4230)$ \\
Birth height $(\mathrm{cm})$ & 47 & $51(45-56)$ \\
Birth head circumference $(\mathrm{cm})$ & 48 & $34(29-53)$ \\
\hline
\end{tabular}

BMI=body mass index; $\mathrm{N}=$ numbers

Table 3. Intrasurgical data.

\begin{tabular}{lllllll}
\hline Suture & \multicolumn{2}{l}{ Vaginal Mucosa } & \multicolumn{3}{l}{ Perineal Muscle } & Skin transcutaneous \\
\cline { 2 - 7 } technique & $\mathbf{N}$ & $\mathbf{\%}$ & $\mathbf{N}$ & $\mathbf{\%}$ & $\mathbf{N}$ & $\mathbf{( \% )}$ \\
\hline Continuous & 49 & 98 & 44 & 88 & 44 & 88 \\
Interrupted & 1 & 2 & 6 & 12 & 6 & 12 \\
\hline
\end{tabular}

\subsection{Outcome Parameter}

Intrasurgical thread handling was rated predominantly very good to excellent throughout in all aspects of the assessment, figure 2 .

\section{Handling}

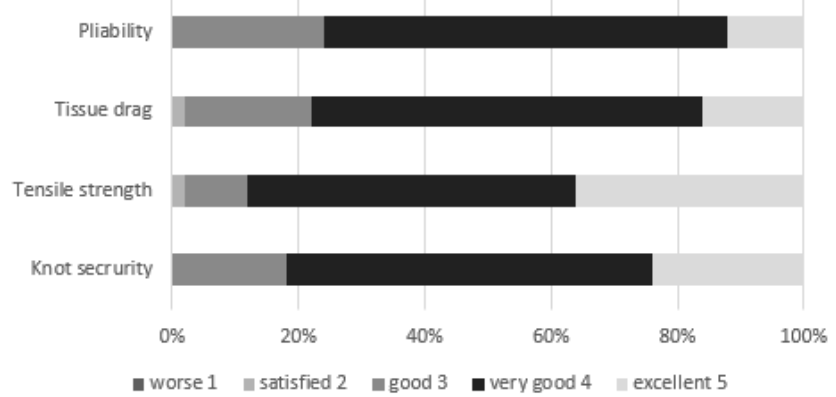

Figure 2. Intrasurgically handling of the suture using a 5-point Likert scale

\subsection{Perineal Pain}

As shown in table 3, more women reported perineal pain during sitting, walking and urinating at $24-48 \mathrm{~h}$ post-partum. In accordance with these findings, the highest pain levels were also observed in the same position, table 4 . Only nine women reported no pain. Eight weeks after delivery, 37 women were pain- free and the pain rates as well as the pain levels strongly decrease in all categories from discharge to eight weeks post-partum table 4 . Comparison with $95 \%$ CI of the literature showed lower short-term perineal pain at 24-48 compared to our suture group. Further classification of perineal pain into different categories indicated significant higher pain rates during repose and urinating in the current population and comparable pain rates during sitting, walking and defecating. Long-term perineal pain at eight weeks postpartum was higher in the present study than the outcome at 3 months after delivery in the literature control group.

Table 4. Short-term and long-term perineal pain rates and pain levels in different position.

\begin{tabular}{lclll}
\hline & Ratio & 95\% CI & Mean & StdDev \\
\hline 24 - 48h & & & & \\
Perineal pain & $38 / 47$ & $0.81[0.67 ; 0.90]$ & & \\
$\quad$ Category & & & & \\
$\quad$ Respose & $29 / 47$ & $0.62[0.47 ; 0.74]$ & 10.38 & 16.47 \\
$\quad$ Sitting & $38 / 47$ & $0.81[0.67 ; 0.90]$ & 30.40 & 28.14 \\
Walking & $37 / 47$ & $0.79[0.65 ; 0.88]$ & 24.62 & 26.59 \\
$\quad$ Urinating & $33 / 46$ & $0.72[0.57 ; 0.83]$ & 16.48 & 23.51 \\
$\quad$ Defecating & $10 / 22$ & $0.45[0.27 ; 0.65]$ & 15.73 & 25.43 \\
Eight weeks \pm 2 weeks & & & \\
Perineal pain & $11 / 48$ & $0.23[0.13 ; 0.37]$ & & \\
$\quad$ Category & & & & \\
In repose & $8 / 48$ & $0.17[0.08 ; 0.30]$ & 2.04 & 6.40 \\
Sitting & $10 / 48$ & $0.21[0.12 ; 0.34]$ & 5.15 & 12.85 \\
Walking & $10 / 48$ & $0.21[0.12 ; 0.34]$ & 5.44 & 13.79 \\
$\quad$ Urinating & $5 / 48$ & $0.10[0.04 ; 0.23]$ & 1.92 & 7.62 \\
Defecating & $5 / 48$ & $0.10[0.04 ; 0.23]$ & 3.08 & 10.32 \\
\hline
\end{tabular}

\subsection{Patient's Satisfaction and Sexual Intercourse}

Patient's satisfaction with the episiotomy repair was high and increases from discharge to eight weeks post-partum (VAS score $84.4 \pm 18.97$ and $91.2 \pm 16.86$, respectively), no difference was seen compared to the literature data. Our population started sexual intercourse 4-5 weeks after delivery, which was 2-3 weeks earlier than in the literature control group. In the present study $50 \%$ of women had sexual intercourse 10 weeks after perineal repair, figure 3 .

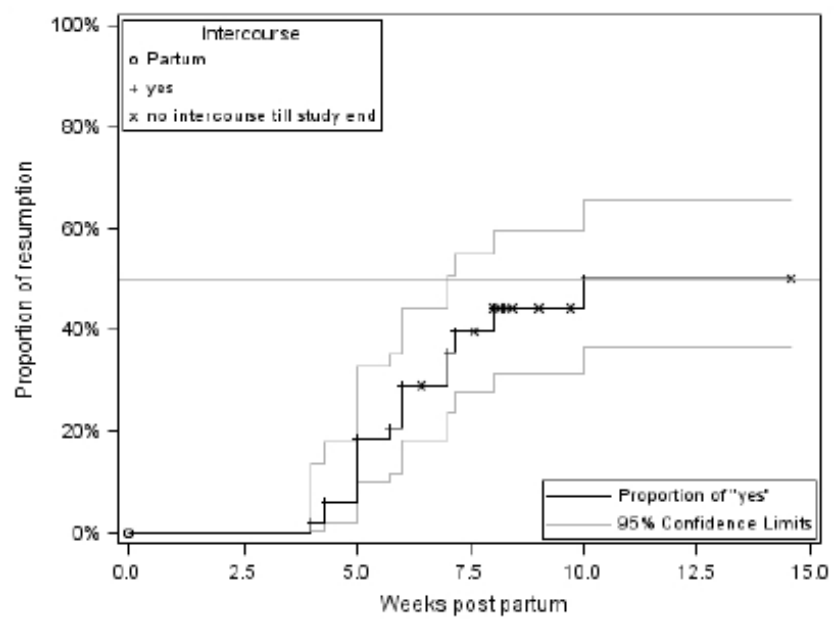

Figure 3. Rates of women resuming post-partum sexual intercourse. 


\subsection{Wound Healing Assessment}

At discharge and eight weeks after delivery professional medical staff assessed the episiotomy wound using the VAS scale with $89.3 \pm 17.4$ and $95.83 \pm 17.4$ respectively, indicating an excellent perineum healing outcome.

\subsection{Postsurgical Complications}

In contrast to $95 \%$ confidence intervals of the literature used for comparison, we observed neither wound dehiscence nor wound infection or delayed wound healing eight weeks after delivery. In addition, no re-suturing of the perineum was necessary and no suture removal due to non-absorbed threads was required.

\section{Discussion}

We report for the first time data on the clinical performance of a novel quick absorbable monofilament suture (Monosyn ${ }^{\circledR}$ Quick) used to close episiotomies after vaginal birth. A large body of literature exists that investigated the outcome of different kind of suture materials and suture techniques for perineal repair. Women's physical, psychological and social well-being is influence by perineal trauma in short-term and long-term post-partum, therefore it is disappointing that the available clinical evidence is lacking high-quality [4].

A Meta-analysis performed in 2007 comparing the interrupted suture technique with the continuous suture technique found less perineal pain in the short term period ( $48 \mathrm{~h}$ to 10 days) and reduced analgesics use with the continuous technique [8]. Kettle et al. also demonstrated in a further meta-analysis that the application of synthetic absorbable suture is more effective in reducing short-term perineal pain, wound dehiscence, wound infection and resuturing compared to catgut. Whereas, suture removal was significantly increased using absorbable synthetic suture for perineal repair in comparison to catgut [6]. A randomised controlled trial analysing rapid, absorbable polyglactin 910 versus standard polyglactin 910 reported less analgesics use and less perineal pain until 10 days post-partum. In addition, suture removal rate was lower with the quicker absorbable suture variant, but wound gaping was more often observed compared with the standard suture material [7].

We compared our findings with the published data available for fast-absorbable, polyglactin 910 multiflament (Vicryl ${ }^{\circledR}$ Rapide), because this suture type is commonly used in gynaecological centres and in addition, researchers believe that fast-absorbing polygalctin should be chosen as first-line suture material for episiotomy closure [15].

In accordance with the literature which indicates that the continuous suture technique should be the method of choice for perineum repair, because it offers several advantages compared to the interrupted suture technique, also in the present study the fast-absorbing monofilament suture was applied in the continuous suture technique to approximate the vaginal mucosa, the perineal muscle and the skin.
Intrasurgical handling of the thread was assessed in different categories by several obstretricians. Results prove an overall very good to excellent handling for episiotomy closure. Wound healing complications after the application for the novel quick absorbable monofilament was $0 \%$ in our study, which differs clearly from the used $95 \%$ CI of the literature data for wound dehiscence, re-suturing, suture removal and remaining sutures seen with fast-absorbing polyglactin multifilaments. Based on that, overall opinion of the obstretrician in regard to perineal wound healing rated using a VAS scale ( 0 worst - 100 excellent) at discharge and eight weeks post-partum was $89.3 \pm 17.4$ and $95.83 \pm 17.4$, indicating that the usage of the new suture permits an excellent wound healing outcome.

In line with the literature data, we observed high patient satisfaction with the episiotomy repair in the short-term and long-term follow up. In our population $50 \%$ of women had resumed intercourse 10 weeks after delivery and started sexual intercourse 2-3 weeks earlier compared to the literature control group (4-5 weeks vs. seven weeks after delivery, respectively).

Perineal pain assessment using the VAS scale (0 low - 100 high) showed a strong decrease of perineal pain rates as well as pain scores from discharge to eight weeks post-partum in our group $(81 \% ; 23 \%$, respectively). Length of hospital stay was about 2 days in the current study. Therefore, perineal pain reported at discharge was compared to the $95 \% \mathrm{CI}$ of the literature control group reported at $24-48 \mathrm{~h}$. In addition, a comparison of our findings in regard to perineal pain at eight weeks after delivery was performed to the $95 \%$ CI of the historical literature group seen at 3 months after delivery, because results evaluated at eight weeks were rare. Shortterm and long-term perineal pain rates as well as pain levels were higher using the new quick-absorbable monofilament suture than with a rapidly absorbable, braided polyglactin 910 suture. This observation could be due to the fact that the novel quick-absorbable, monofilament suture (Monosyn ${ }^{\circledR}$ Quick) gives longer wound support as the rapidly-absorbing, multifilament polyglactin 910 (Vicryl ${ }^{\circledR}$ Rapide) applied in the historical literature control group, (14 - 21 days vs. 14 days after implantation, respectively). In addition, Vicryl ${ }^{\circledR}$ Rapide is completely absorbed after 28 days compared to 56 days in the case of Monosyn ${ }^{\circledR}$ Quick suture. This fact could be the reason for longer and higher perineal pain observed in our study.

To date only 2 RCTs evaluating a monofilament absorbable suture $\left(\right.$ Monocryl ${ }^{\circledR}$ ) versus a fast-absorbable, braided, multifilament (Vicryl ${ }^{\circledR}$ Rapide) for episiotomy repair have been published [12, 15]. These sutures differ substantially in their properties. Based on their smaller surface, monofilament sutures cause minimal tissue reaction [12]. Monofilaments consisting of polyglycolide-cocaprolactone (e.g. Monocryl ${ }^{\circledR}$ ) carry $25 \%$ of the original tensile strength after 14 days and complete absorption is seen within 120 days. In contrast, fast-absorbing, polyglactin (Vicryl ${ }^{\circledR}$ Rapide) has due to its braided structure a larger surface and niches in which bacteria cannot be easily reached 
by immune cells of the immune system [12]. This suture material is completely absorbed in 42 days and no tensile strength is present after 14 days. The novel quick- absorbable monofilament suture (Monosyn ${ }^{\circledR}$ Quick) is a triblock copolymer made of glycolide $(72 \%)$, $\varepsilon$-caprolactone $(14 \%)$ and trimethylene carbonate (14\%). After implantation this suture possess $70-80 \%$ of its initial tensile strength after 5 days and $20-30 \%$ after 10 days. Tensile strength is completely lost after 14-21 days and the suture mass absorption is ensued after 56 days.

The MOVE-trial comparing Vicryl ${ }^{\circledR}$ Rapide versus Monocryl $^{\circledR}$ found equal pain rates, and no difference regarding analgesics use and proportions of women starting sexual intercourse [12]. But significantly less skin dehiscences were reported in the Monocryl ${ }^{\circledR}$ group at different time points ( $8 \%$ vs. $22 \%$ at 10 days; $10 \%$ vs. $25 \%$ after 3 months; respectively). In addition, the authors of the MOVE-trial mentioned that wound infections and suture removals occurred in both groups, but with no significant difference. These findings varied from our's, because neither wound infections nor wound dehiscences were observed and suture removals were not necessary.

The second RCT performed by Kokanali and co-workers investigated Vicryl $^{\circledR}$ Rapide versus Monocryl ${ }^{\circledR}$ in combination with either the continuous or the interrupted suture technique regarding wound healing, perineal pain in different positions at $24 \mathrm{~h}$ and 10 days, and the occurrence of complications [15]. Pain scores were equivalent in both suture groups, but significantly higher with the interrupted technique as with the continuous suture technique, maybe because of a higher tension in the interrupted suture. No wound infections or haemotomas were observed. Incomplete wound healing was present in both suture groups and ranges from $5-7.5 \%$ and residual sutures were also noted, $2.5 \%$ with Monocryl ${ }^{\circledR}$ and $5 \%$ with Vicryl ${ }^{\circledR}$ Rapide. After 6 weeks $25-27 \%$ of women had resumed sexual intercourse. Comparison of our pain score at $24 \mathrm{~h}$ and eight weeks are comparable with the outcome shown in the MOVE-trial and the RCT performed by Kokanali et al. Furthermore, the rate of women reporting sexual intercourse at 6 weeks in the MOVE-trial and in the RCT of Kokanali et al. were congruent with our results. In agreement with Kokanali et al., we found no wound infection and haematoma, but in contrast to them our findings showed no incomplete wound healing and suture removal was also not necessary.

Concordant with the authors of the MOVE-trial we raised the question whether a rapidly-absorbable multifilament suture is still the optimal choice for perineal repair, as suggested in the guidelines [12].

Limitation of the study is the single centre design including a small sample size with a short term follow up until eight weeks and a comparison to historical data.

\section{Conclusion}

Based on the current findings, we conclude that the novel quick-absorbable monofilament suture provides an excellent suture choice for episiotomy closure. Further well-designed randomized controlled trials comparing a rapidly-absorbable multifilament with a quick-absorbable monofilament suture should be performed to increase the clinical evidence in regard to the best suture depending on its filament configuration and degradation profile.

\section{Author Contribution}

Dr. Petra Baumann conception, design, analysis and interpretation of data, drafting of the work, substantial editing, approval of the final version, agreeing to be accountable for all aspects of the work.

Prof. Weigel interpretation of data, substantial editing, approval of the final version, agreeing to be accountable for all aspects of the work.

\section{Financial Support and Conflicts of Interest}

B. Braun Surgical S. A. sponsored and funded the study. The Medical Scientific Affairs department of Aesculap AG was responsible for project management, data management, monitoring, statistics, and study registration. $\mathrm{PB}$ is an employee of Aesculap AG and received salary during the conducting of the study. B. Braun Surgical S. A. reimbursed the article processing charge. The other authors declare no conflicts of interest.

\section{Acknowledgements}

The authors would like to thank all participating women, as well as the caring nurse and obstretricians in the Leopoldina Hospital Schweinfurt, Department of Gynaecology, Schweinfurt, Germany. The authors would like to gratefully acknowledge Viktor Breul, M. Sc., Biomedical Statistician, Aesculap AG, Medical Scientific Affairs, Tuttlingen, Germany, for the statistical analysis.

\section{References}

[1] Weber AM, Meyn L. Episiotomy us in the United States, 1979-1997. Obstet Gynaecol 2002; 100: 1177-82.

[2] De Leeuw JW, Kuijken JP, Bruinse HW. Mediolateral episiotomy reduces the risk for anal sphincter injury during surgical vaginal delivery. BJOG 2008, 115: 104-8.

[3] Christianes WNM, de Vries R. Trends in the medicalization of childbirth in Flanders and the NetherlandsMidwifery. 2013; 29: e1-8.

[4] Frohlich J, Kettle C. Perineal care. BMJ Clinical Evidence 2015; 03: 1401 .

[5] Sleep J. Perineal care, a series of 5 randomized controlled trials. In: Robinson S, Thomson A (eds) Midwives research and childbirth. Chapman and Hall, London, pp 199-251. 
[6] Kettle C, Johanson RB. Absorbable synthetic versus catgut suture material for perineal repair. Cochrane Database Syst Rev. 2000; (2): CD000006.

[7] Kettle C, Hills RK, Jones P, Darby L, Gray R, Johanson R. Continuous versus interrupted perineal repair with standard or rapidly absorbed sutures after spontaneous vaginal birth: a randomised controlled trial. Lancet. 2002; 359 (9325): 221723.

[8] Kettle C, Dowswell T, Ismail KM. Continuous and interrupted suturing techniques for repair of episiotomy or second-degree tears. Cochrane Database Syst Rev. 2012 Nov 14; 11: CD000947.

[9] Kebaili S, Trigui K, Louati D, Smawi M, Hlima S, Dammak A, Gassara H, Chaabane K, Amouri H. Continuous versus interrupted techniques for repair of mediolateral episiotomy: a prospective 6 months follow-up study. Androl Gynecol: Curr Res. 2013; 1: 4.

[10] Morano S, Mistrangelo E, Pastorino D, Lijoi D, Costantini S, Ragni N. A randomized comparison of suturing techniques for episiotomy and laceration repair after spontaneous vaginal birth. J Minim Invasive Gynecol. 2006; 13 (5): 457-62.

[11] Kettle C, Dowswell T, Ismail KM. Absorbable suture materials for primary repair of episiotomy and second degree tears. Cochrane Database Syst Rev. 2010 Jun 16; (6): CD000006.

[12] Odijk R, Hennipman B, Rousian M, Madani K, Dijksterhuis M, de Leeuw JW, van Hof A. The MOVE-trial: Monocryl ${ }^{\circledR}$ vs. Vicryl Rapide ${ }^{\mathrm{TM}}$ for skin repair in mediolateral episiotomies: a randomized controlled trial. BMC Pregnancy Childbirth. 2017 Oct 16; 17 (1): 355.

[13] Dandolu V, Chatwani A. Randomized comparison of chromic versus fast-absorbing polyglactin 910 for post-partum perineal repair. Obstet Gynecol. 2004 Nov; 104 (5 Pt 1): 1104.
[14] Bharathi A, Reddy DB, Kote GS. A prospective randomized comparative study of Vicryl Rapide versus chromic catgut for episiotomy repair. J Clin Diagn Res. 2013; 7 (2): 326-30.

[15] Kokanali, Ugur M, Kuntay Kokanalı M, Karayalcın R, Tonguc E. Continuous versus interrupted episiotomy repair with monofilament or multifilament absorbed suture materials: a randomised controlled trial. Arch Gynecol Obstet. 2011; 284 (2): $275-80$

[16] Leroux N, Bujold E. Impact of chromic catgut versus polyglactin 910 versus fast-absorbing polyglactin 910 sutures for perineal repair: a randomized, controlled trial Am J Obstet Gynecol. 2006; 194 (6): 1585-90.

[17] McElhinney BR, Glenn DR, Dornan G, Harper MA. Episiotomy repair: Vicryl versus Vicryl Rapide. Ulster Med J. 2000; 69 (1): 27-9.

[18] Greenberg JA, Lieberman E, Cohen AP, Ecker JL. Randomized comparison of chromic versus fast-absorbing polyglactin 910 for post-partum perineal repair. Obstet Gynecol. 2004; 103 (6): 1308-13.

[19] Valenzuela P, Saiz Puente MS, Valero JL, Azorín R, Ortega R, Guijarro R. Continuous versus interrupted sutures for repair of episiotomy or second-degree perineal tears: a randomised controlled trial. BJOG. 2009; 116 (3): 436-41.

[20] Wong T, Wak HL, Wong HK, Leung KY. Perineal repair with standard versus rapidly adsorbed sutures after vaginal birth: a randomized controlled trial. Hong Kong J Gynaecol Obstet Midwifery 2006; 6: 4-9.

[21] Dencker A, Lundgren I, Sporrong T. Suturing after childbirth-a randomised controlled study testing a new monofilament material. BJOG. 2006 Jan; 113 (1): 114-6. 